\section{Carpological Variability of Almond [Prunus dulcis (Mill.) D.A. Webb cv. Nonpareil] in a Single Orchard during Seven Consecutive Harvests}

\author{
Craig A. Ledbetter ${ }^{1}$ and Mark S. Sisterson \\ U.S. Department of Agriculture, Agricultural Research Service, San Joaquin \\ Valley Agricultural Sciences Center, Crop Diseases, Pests \& Genetics \\ Research Unit, 9611 S. Riverbend Avenue, Parlier, CA 93648-9757
}

Additional index words. dimensional analysis, evaluation, regression, varietal standard

\begin{abstract}
A multiyear study was conducted in California's San Joaquin Valley to examine variability of carpological characteristics of the popular Nonpareil almond cultivar. Samples of 'Nonpareil' almond fruit were collected from a single orchard during seven consecutive harvests and evaluated for 19 specific carpological characters. Harvest year significantly affected all measured variables. Of the evaluated characters, fruit weight was the most variable between years, whereas kernel percentage was one of the least variable characters. As a result of year-to-year variability in carpological characters, the relationship between kernel weight and all other measured variables was investigated to determine if regression models could better describe kernels. Kernel weight best explained variability in kernel surface area $\left(R^{2}=0.943\right)$ with year-to-year variability having only a minor affect on the relationship. The results indicate that 'Nonpareil' almonds cannot be described using simple metrics and that definitive metrics must be able to account for differences among years.
\end{abstract}

Dozens of cultivars have contributed to California's almond production, but the Nonpareil cultivar, selected in 1879 , has consistently dominated California almond tonnage as a result of its excellent kernel characteristics and horticultural qualities. Currently, 'Nonpareil' occupies $37 \%$ of California almond acreage (Anon., 2009) and is marketed in an exclusive category that accounted for over 276 million kilograms of the total 732 million kilogram harvest during 2008 (Anon., 2009).

Although California's almond marketing categories (Nonpareil, Carmel, California, Mission, NePlus Ultra, and In-shell) group cultivars having similar kernel shape and appearance to facilitate their sale and use, no objective varietal standards exist to assign cultivars to a given category. Handlers, responsible for marketing almonds, have leeway in cultivar placement within specific marketing categories (Asaii et al., 1996). Consequently, cultivars other than 'Nonpareil' (e.g., 'Jeffries', 'Kapareil', and 'Milow') have at times been included within the Nonpareil marketing category. Public and private breeding efforts are now providing new cultivars for California orchards with their future marketing category still uncertain. Objective varietal standards for the specific marketing categories would benefit

\footnotetext{
Received for publication 10 Aug. 2010. Accepted for publication 19 Oct. 2010.

We sincerely thank Biological Technician Sharon J. Peterson for her assistance in data collection during the study.

${ }^{1}$ To whom reprint requests should be addressed; e-mail Craig.Ledbetter@ars.usda.gov.
}

nurseries and almond producers in providing information on new cultivars during the decision-making process before orchard establishment.

Almonds in the Nonpareil category are a preferred choice in applications where the kernel is prominently visible and have historically received premium prices compared with other marketing categories (Wood, 1937). The Nonpareil cultivar has been categorized based on its free amino acid composition and fatty acid profile (García-López et al., 1996; Martín-Carratalá et al., 2002). Similarly, genetic markers have been developed to distinguish 'Nonpareil' from other cultivars (Dangl et al., 2009). However, marketing categories separate almond cultivars on the basis of kernel shape and appearance, and no standards to distinguish 'Nonpareil' kernels from those of other cultivars currently exist.

Interannual variability of phenological and carpological traits has been studied previously in a breeding population of almond (SánchezPérez et al., 2007), and population mean values of several carpological traits (nut weight, kernel weight, kernel thickness) were found to vary significantly among the 4 harvest years. To our knowledge, studies have not yet been conducted to document the extent of interannual variability of carpological characters of the important 'Nonpareil' almond. Because 'Nonpareil' buyers pay a premium for its specific kernel characteristics, a more precise characterization of how 'Nonpareil' kernels vary between harvest years would benefit the industry. Hence, our objective in the current study is to document the variability in appearance, shape, and carpological characteristics of 'Nonpareil' almond kernels during seven consecutive growing seasons.

\section{Materials and Methods}

Plant materials. Virus-free dormant budsticks of 'Nonpareil' almond (IR No. 0015-1) were received from the National Research Support Project 5 in Prosser, WA, during the winter of 1993-1994. Budsticks were cleftgrafted on 1-year-old 'Nemaguard' seedling peach rootstocks previously established in the research orchard at the San Joaquin Valley Agricultural Sciences Center in Parlier, CA. Ten 'Nonpareil' trees were established as a control cultivar in a variety trial for promising almond selections from the Agricultural Research Service (ARS) almond breeding effort. Trees were maintained using standard orchard procedures and cultural practices. Irrigation, fertilization, and insecticides were administered as needed to maintain an optimal environment for plant growth. At no time during the life of the trees has non-infectious bud failure ever been observed. Trees of ARS almond selections and pollen-compatible cultivars (Mission, Monterey, and NePlus) were available throughout the orchard to provide the needed pollination to ensure adequate nut set.

'Nonpareil' trees were in their tenth leaf (2003) during the first year of seven consecutive harvests. At each harvest period, nuts were manually removed from trees by knocking after hull split had progressed to at least $80 \%$. Almond fruits were collected from each tree on polypropylene tarps and pooled together between tree rows for a 3- to 6-d period of sun drying. A random sample of whole almond fruit (hull intact) was then collected and frozen for subsequent analysis.

Sample preparation and analyses. Before laboratory analysis, the frozen samples were dried for $\approx 18 \mathrm{~h}$ at $45^{\circ} \mathrm{C}$. Dried almond fruits were characterized as follows:

- Gravimetric analysis of whole fruit, nut, and kernel for each intact fruit;

- Calculation of hull percentage [(hull wt/fruit wt $\times 100]$ and kernel percentage [(kernel wt $/$ nut wt) $\times 100$ ];

- Measurements of kernel length, width, and thickness; and

- Measurement of pellicle color coordinates luminosity, chroma, and hue.

Both insect-damaged kernels and doublekernelled nuts were excluded from the study. Fruit, nut, and hull weights were acquired on an analytical balance (American Scientific Products, Columbus, $\mathrm{OH}$ ) with an accuracy of $0.0001 \mathrm{~g}$. A digital caliper (Starrett, Athol, MA) was used for kernel dimensional measurements (accuracy to $0.01 \mathrm{~mm}$ ). Pellicle color was measured with a Minolta Chroma Meter CR-200 (Minolta Camera Co. Ltd., Osaka, Japan) equipped with an 8-mm measurement aperture. The measuring head of the CR-200 uses diffuse illumination of a xenon arc lamp pulse within a mixing chamber to obtain color coordinate values from 
non-uniform sample surfaces. After calibration of the CR-200 to a standard white tile (calibration plate CR-A43), the meter was programmed to obtain color properties luminosity, chroma, and hue.

Kernel dimensional ratios length:width, length:thickness, and width:thickness were calculated for each kernel. To further characterize 'Nonpareil' kernels, geometric mean diameter, sphericity, surface area, and kernel volume were calculated from formulas published previously (Özgüven and Vursavuş, 2005):

- Geometric mean diameter: $=(\mathrm{LWT})^{1 / 3}$

- Sphericity $=\left[(\mathrm{LWT})^{1 / 3} / \mathrm{L}\right] 100$

- Surface area $=\pi\left(\mathrm{LWT}^{1 / 3}\right)^{2}$

- Volume $=\left(\pi \mathrm{B}^{2} \mathrm{~L}^{2}\right) /[6(2 \mathrm{~L}-\mathrm{B})]$ where $\mathrm{B}=$ $(\mathrm{WT})^{0.5}$

Kernel density was then calculated from each known kernel weight and kernel volume:

- Density $=$ kernel weight/kernel volume

Estimating minimum sample size. Sample sizes varied between years with the smallest number of almonds examined in 2003 ( $\mathrm{n}=$ 30 ) and the largest number examined in 2008 $(\mathrm{n}=862)$. As a result of variation in sample size, the minimum sample size required to accurately estimate the mean and variance of each measure was investigated. Establishing appropriate sample sizes will aid in determining the validity of comparing results between years in our study and will provide estimates for future studies that examine kernel characteristics.

To determine the effect of sample size on accurately estimating the mean and variance of each measure, a resampling procedure (Manly, 1991) was imposed on the 2008 harvest data. Specifically, a random sample of two to 50 almonds was drawn from the 2008 harvest data and the mean and variance of each measure determined. This procedure was repeated 5000 times for each sample size and $95 \%$ confidence intervals for means and variances were obtained. Confidence intervals were plotted against sample size to determine the sample size at which the upper and lower bounds of the $95 \%$ confidence interval reached an asymptote.

Statistical analyses. Data were tabulated and analyzed using JMP stats (Version 7; SAS, Cary, NC). Analysis of variance (ANOVA) was used to test the effect of harvest year on all variables. Before performing ANOVAs, data were subjected to tests ensuring variance homogeneity. Linear regressions were conducted on specific variable pairs in each harvest year and slopes were compared among harvest years.

\section{Results}

Estimating minimum sample size. The upper and lower bounds of the 95\% confidence intervals for the mean and variance of each measure subjected to the resampling procedure reached an asymptote between a sample size of 20 and 30. Fruit weight and kernel percentage were chosen to illustrate this prop- erty (Fig. 1). Fruit weight was one of the most variable measures within ( $\mathrm{CV}$ for $2008=13.2$ ) and between years ( $\mathrm{CV}$ between years $=18.5$ ). In contrast, kernel percentage was one of the least variable measures within (CV for $2008=$ 5.6) and between years ( $\mathrm{CV}=7.43)$.

Fruit, nut, and kernel weight and variability in kernel dimensions. All measured variables were significantly affected by harvest year (Table 1). Over 7 years of data col- lection, measures of fruit, nut, and kernel weight varied from 1.36-fold (nut weight) to 1.46-fold (fruit weight). The greatest fruit, nut, and kernel weights were observed in the same year (2005), but the lowest values for each of these measures was observed in different years (Table 1). Observed variability in kernel weights could have an economic impact on 'Nonpareil' growers because 2005 kernels averaged 70 kernels/100 g (18/20
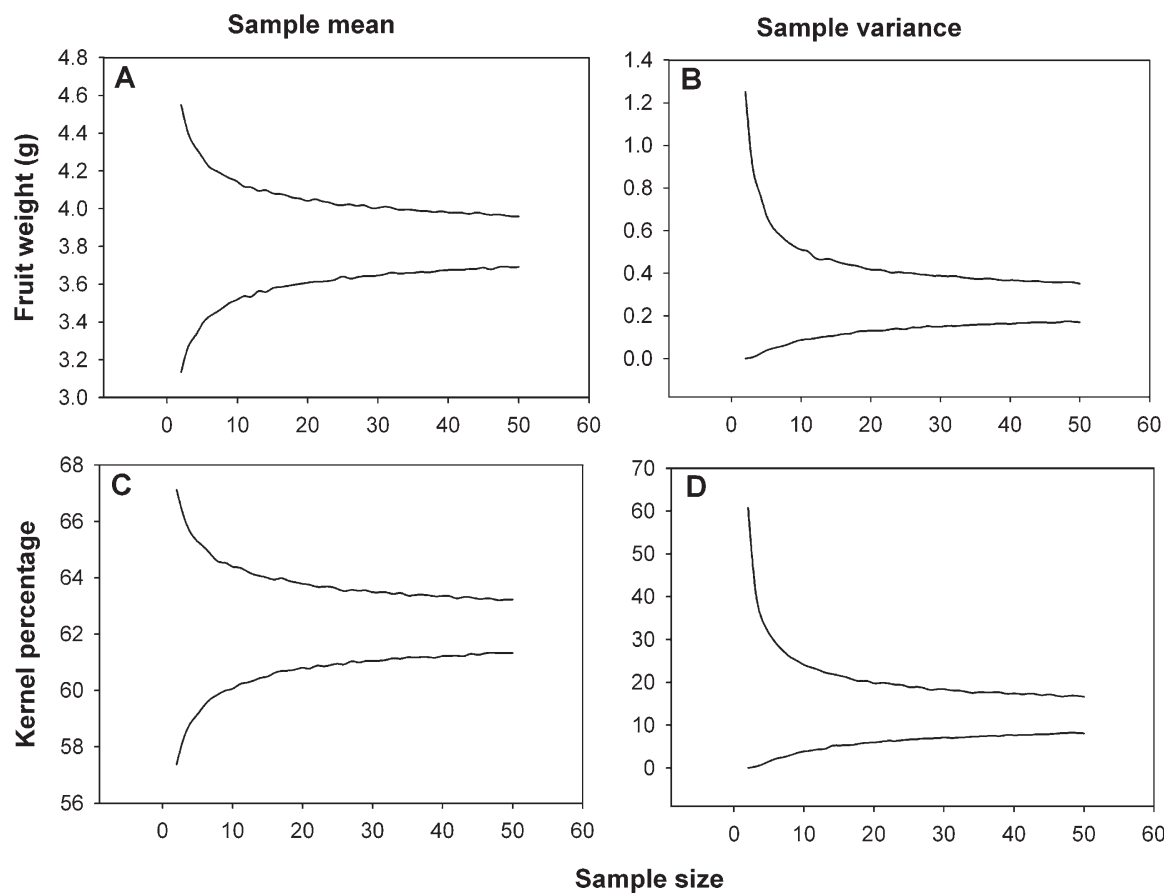

Fig. 1. Confidence intervals $(95 \%)$ to estimate minimum adequate sample sizes for fruit weight $(\mathbf{A}=$ sample mean, $\mathbf{B}=$ sample variance $)$ and kernel percentage $(\mathbf{C}=$ sample mean, $\mathbf{D}=$ sample variance $)$. Plots are based on 2008 harvest data $(n=862)$ with 5000 random samples (case resampling) at each sample size.

Table 1. Variability of evaluated carpological characteristics for 'Nonpareil' almonds from a single orchard harvested in Parlier, CA, 2003 through 2009.

\begin{tabular}{lccrr}
\hline & & \multicolumn{2}{c}{ Annual mean range $(\mathrm{yr})^{\mathrm{y}}$} \\
\cline { 3 - 5 } Variable & Grand mean $\pm \mathrm{sD}^{\mathrm{z}}$ & $\begin{array}{c}\text { Mean confidence } \\
\text { interval }\end{array}$ & \multicolumn{1}{c}{ Minimum } & \multicolumn{1}{c}{ Maximum } \\
\hline Fruit weight $(\mathrm{g})^{* * x}$ & $4.17 \pm 0.77$ & $4.14-4.20$ & $3.77(2006)$ & $5.48(2005)$ \\
Nut weight $(\mathrm{g})^{* *}$ & $1.80 \pm 0.26$ & $1.78-1.81$ & $1.66(2008)$ & $2.25(2005)$ \\
Kernel weight $(\mathrm{g})$ * $^{* *}$ & $1.16 \pm 0.18$ & $1.15-1.16$ & $1.04(2008)$ & $1.43(2005)$ \\
Hull percentage $(\%)^{* *}$ & $56.52 \pm 3.96$ & $56.36-56.68$ & $51.76(2004)$ & $59.31(2009)$ \\
Kernel percentage (\%)** & $64.40 \pm 4.78$ & $64.21-64.60$ & $61.74(2004)$ & $67.67(2009)$ \\
Kernel length $(\mathrm{mm})^{* *}$ & $23.94 \pm 1.80$ & $23.86-24.01$ & $23.46(2008)$ & $26.70(2005)$ \\
Kernel width $(\mathrm{mm})^{* *}$ & $12.75 \pm 0.90$ & $12.72-12.79$ & $12.11(2004)$ & $13.59(2009)$ \\
Kernel thickness (mm) & $7.98 \pm 0.51$ & $7.96-8.00$ & $7.67(2008)$ & $8.46(2006)$ \\
Kernel L:W ratio** & $1.88 \pm 0.11$ & $1.87-1.88$ & $1.73(2006)$ & $1.99(2004)$ \\
Kernel L:T ratio** & $3.01 \pm 0.29$ & $3.00-3.02$ & $2.59(2006)$ & $3.38(2005)$ \\
Kernel W:T ratio** & $1.60 \pm 0.14$ & $1.59-1.61$ & $1.49(2006)$ & $1.74(2005)$ \\
Pellicle Luminosity** & $52.66 \pm 4.66$ & $52.48-52.85$ & $44.5(2003)$ & $57.1(2004)$ \\
Pellicle Chroma** & $38.74 \pm 2.84$ & $38.63-38.86$ & $34.4(2003)$ & $40.7(2005)$ \\
Pellicle hue** & $69.43 \pm 2.61$ & $69.32-69.54$ & $67.6(2003)$ & $70.8(2006)$ \\
Geometric mean $(\mathrm{mm})^{* *}$ & $13.44 \pm 0.68$ & $13.41-13.46$ & $13.01(2008)$ & $14.28(2005)$ \\
Sphericity $(\%)^{* *}$ & $56.28 \pm 2.69$ & $56.18-56.39$ & $53.55(2005)$ & $60.75(2006)$ \\
Surface area $\left(\mathrm{mm}^{2}\right)^{* *}$ & $568.74 \pm 58.37$ & $566.38-571.10$ & $532.60(2008)$ & $641.15(2005)$ \\
Volume $\left(\mathrm{mm}^{3}\right)^{* *}$ & $811.59 \pm 123.22$ & $806.61-816.58$ & $730.36(2008)$ & $951.37(2005)$ \\
Density $\left(\mathrm{g} \cdot \mathrm{cm}^{-3}\right)^{* *}$ & $1.42 \pm 0.06$ & $1.421-1.426$ & $1.39(2007)$ & $1.51(2005)$ \\
\hline
\end{tabular}

${ }^{7}$ Grand mean was calculated from combined data of all seven harvests $(n=2347)$.

${ }^{y}$ Annual means were calculated from a variable number of fruit depending on year $(2003=30,2004=124$, $2005=157,2006=339,2007=343,2008=862,2009=492$ )

${ }^{\mathrm{x}}$ Significant $(P<0.01)$ difference in variable among examined harvest years as determined through analysis of variance $(d f=6,2346)$.

$\mathrm{L}: \mathrm{W}=$ length:width; $\mathrm{L}: \mathrm{T}=$ length:thickness; $\mathrm{W}: \mathrm{T}$ = width:thickness. 
count/ounce) whereas kernels produced in 2008 averaged 96 kernels/100 g (27/30 count/ ounce category), limiting the latter's usefulness in some processing applications. Variability ratios for hull and kernel percentages were lower than for measures of fruit, nut, and kernel weights. Across seven harvests, kernel percentage averaged $64.4 \%$ with annual means ranging from $61.7 \%$ (2004) to $67.7 \%$ (2009). Minimum (51.8\%) and maximum (59.3\%) annual means for hull percentage were also recorded in these same years.

Mirroring fruit, nut, and kernel weight, kernel length was greatest in 2005, but kernels were widest in $2009(13.6 \mathrm{~mm})$ and thickest in 2006 (Table 1). Kernel dimensional ratios were lowest in 2006 and at maximums in 2004 (length:width ratio) and 2005 (length: thickness, width:thickness ratios). Of the kernel dimensional ratios, kernel length:thickness was the most variable between years. Kernel dimensional ratios obtained in a previous study (Ledbetter and Palmquist, 2006a) with 'Nonpareil' kernels (length: width $=1.85$, length:thickness $=2.85$, width thickness $=1.54$ ) were similar to the values reported here.

Data from the 7 harvest years were pooled to examine correlations between fruit, nut, and kernel weights and kernel dimensions. Correlation coefficients of 0.843 and higher were observed for all pairwise comparisons among fruit, nut, and kernel weights (Table 2 ). The highest correlation between kernel weight and a kernel dimensional value was for width (0.866), whereas the lowest was between kernel weight and kernel thickness (0.436). Correlation values among kernel dimensions were more variable. Kernel lengths and widths correlated to a much higher degree (0.658) as compared with length/thickness $(-0.002)$ or width/thickness $(0.213)$, in agreement with reports by Kester and Asay (1975) on the general relationship between kernel size and shape. Spiegel-Roy and Kochba (1981) examined correlations between nut and kernel characteristics of over 2000 seedlings from 19 genetically diverse almond progenies. In some cases, correlation coefficients reported here were similar to reports by Spiegel-Roy and Kochba (1981) (kernel width and thickness, 0.20 versus 0.213 ; kernel thickness and nut weight, 0.16 versus 0.259 ), and in other pairwise comparisons, coefficients were surprisingly different (kernel length and kernel width, -0.16 versus 0.658 ; kernel length and nut weight, -0.08 versus 0.800 ; kernel weight and nut weight, 0.26 versus 0.884 ).

Pellicle color variability. Pellicle color was described with color properties luminosity, chroma, and hue. All three coordinates varied significantly across harvest years (Table 1). In 2003, kernel pellicles were darker than in other harvest years as evidenced by minimal values for all three coordinates in that harvest year (Table 1). Pellicle darkening has been demonstrated to increase with temperature for almonds held in long-term storage (Ledbetter and Palmquist, 2006b). As such, pellicle color coordinate values reported for 2003 may be the result of unusually high ground temperatures during sun drying. Although color affects a consumer's perception of quality (Clydesdale, 1991), pellicle color is not part of the USDA grading standard for shelled almonds (Anon., 1997).

Multidimensional kernel characterization. The multidimensional kernel characters geometric mean diameter, sphericity, surface area, volume, and density were all significantly affected by harvest year (Table 1). Corresponding with maximum fruit, nut, and kernel weights, almonds harvested in 2005 were significantly larger in kernel geometric mean diameter (14.3 mm), kernel surface area (641.2 $\left.\mathrm{mm}^{2}\right)$, and kernel volume $\left(951.4 \mathrm{~mm}^{3}\right)$ than almonds from other harvests (Table 1). Kernel density was highest in $2005\left(1.51 \mathrm{~g} \cdot \mathrm{cm}^{-3}\right)$ and lowest in $2007\left(1.39 \mathrm{~g} \cdot \mathrm{cm}^{-3}\right)$. Kernel sphericity ranged from $60.75 \%$ (2006) to $53.55 \%$ (2005) with the calculated values comparing favorably with those reported previously by Aktas et al. (2007). Kernel weight correlated well

Table 2. Pearson product-moment correlations for fruit, nut, and kernel weights, kernel dimensions, and multidimensional kernel variables from 7 combined harvest years $(n=2347)$ of 'Nonpareil' almond harvested in Parlier, CA, 2003-2009.

\begin{tabular}{|c|c|c|c|c|c|c|}
\hline & Fruit wt & Nut wt & Kernel wt & $\begin{array}{c}\text { Kernel } \\
\text { length }\end{array}$ & $\begin{array}{l}\text { Kernel } \\
\text { width }\end{array}$ & $\begin{array}{c}\text { Kernel } \\
\text { thickness }\end{array}$ \\
\hline Fruit wt & 1.000 & 0.864 & 0.843 & 0.810 & 0.807 & 0.105 \\
\hline Nut wt & & 1.000 & 0.884 & 0.800 & 0.785 & 0.259 \\
\hline Kernel wt & & & 1.000 & 0.798 & 0.866 & 0.436 \\
\hline Kernel length & & & & 1.000 & 0.658 & -0.002 \\
\hline Kernel width & & & & & 1.000 & 0.213 \\
\hline Kernel thickness & & & & & & 1.000 \\
\hline
\end{tabular}

\begin{tabular}{lccrrr} 
& $\begin{array}{c}\text { Geometric } \\
\text { mean diam }\end{array}$ & Sphericity & $\begin{array}{c}\text { Surface } \\
\text { area }\end{array}$ & Volume & Density \\
\hline Fruit wt & 0.809 & -0.394 & 0.812 & 0.775 & 0.364 \\
Nut wt & 0.860 & -0.327 & 0.861 & 0.832 & 0.320 \\
Kernel wt & 0.969 & -0.204 & 0.971 & 0.960 & 0.303 \\
Kernel length & 0.795 & -0.731 & 0.797 & 0.718 & 0.405 \\
Kernel width & 0.869 & -0.094 & 0.869 & 0.869 & 0.134 \\
Kernel thickness & 0.508 & 0.571 & 0.503 & 0.579 & -0.410 \\
Geometric mean diameter & 1.000 & -0.172 & 0.999 & 0.991 & 0.089 \\
Sphericity & & 1.000 & -0.177 & -0.056 & -0.539 \\
Surface area & & & 1.000 & 0.992 & 0.093 \\
Volume & & & & 1.000 & 0.028 \\
Density & & & & & 1.000 \\
\hline
\end{tabular}

with geometric mean diameter (0.969), kernel surface area (0.971), and kernel volume $(0.960)$ across harvest years (Table 2). Among the gravimetric measures, kernel weight had higher correlations with these multidimensional variables as compared with fruit or nut weights or any of the dimensional measures. Kernel width was most highly correlated with multidimensional variables among the kernel dimensional measures. Low correlations were realized for all gravimetric and dimensional measures with both sphericity and kernel density (Table 2).

Accounting for variability in kernels of different sizes. Linear regressions were constructed to further examine the relationship between kernel weight and kernel dimensional values (length, width, thickness), kernel dimensional ratios (length:width ratio, length: thickness ratio, width:thickness ratio), and multidimensional variables (geometric mean diameter, kernel surface area, kernel volume). Coefficients of determination varied widely, depending on the combination of variables, but were relatively consistent across harvest years (Table 3 ). Kernel dimensional ratios as functions of kernel weight had the lowest coefficients of determination, from $R^{2}=0.00$ for length:width ratio in 2005 to a maximum of $R^{2}=0.123$ for length:thickness ratio in 2006. Compared with dimensional ratios, kernel dimensions regressed as functions of kernel weight yielded higher coefficients of determination. Among the individual dimensions, kernel thickness always had a lower coefficient of determination in any given harvest year as compared with either kernel length or kernel width (Table 3), consistent with a previous study in the same orchard (Ledbetter and Palmquist, 2006a). Multidimensional variables regressed as functions of kernel weight had high coefficients of determination across harvest years, ranging from $R^{2}=0.824$ to $R^{2}=0.944$.

Harvest year significantly affected the relationship between kernel weight and all measures evaluated in regression analyses (Table 3). However, in some of the regression models, the effect of harvest year appears to be minor. For example, we compared regression models with and without the effect of harvest year for regressions of kernel surface area as a function of kernel weight and for regressions of kernel geometric mean diameter as a function of kernel weight (Table 4). Comparison of coefficients of determination in regression models including and excluding harvest year indicated that less than $1.5 \%$ of the variation in kernel surface area or kernel geometric mean diameter was explained by harvest year, whereas at least $94 \%$ of the variation in kernel surface area or kernel geometric mean was explained by kernel weight (Table 4). Accordingly, this suggests that a significant effect of harvest year was detected as a result of our large samples size and that harvest year has only a minor affect on the relationship.

Because regressions of kernel weight on kernel surface area provided the highest coefficients of determination (Table 3 ) and the effect of year on the relationship was minor (Table 4), data from the seven harvests were pooled to identify the specific linear 
Table 3. Coefficients of determination and slope comparisons between harvest years for kernel dimensions, kernel dimensional ratios, and multidimensional variables as functions of kernel weight.

\begin{tabular}{lcccccccrr}
\hline Response variable & 2003 & 2004 & 2005 & 2006 & 2007 & 2008 & 2009 & $F$-ratio & Prob. $>F$ \\
\hline Kernel length & 0.470 & 0.637 & 0.666 & 0.639 & 0.579 & 0.654 & 0.634 & 3.57 & 0.0016 \\
Kernel width & 0.666 & 0.622 & 0.612 & 0.637 & 0.425 & 0.660 & 0.610 & 10.13 & $<0.0001$ \\
Kernel thickness & 0.443 & 0.149 & 0.198 & 0.090 & 0.330 & 0.276 & 0.279 & 4.22 & 0.0003 \\
L:W ratio & 0.064 & 0.009 & 0.000 & 0.002 & 0.016 & 0.022 & 0.003 & 4.81 & $<0.0001$ \\
L:T ratio & 0.018 & 0.091 & 0.081 & 0.123 & 0.004 & 0.032 & 0.046 & 3.31 & 0.003 \\
W:T ratio & 0.002 & 0.078 & 0.071 & 0.119 & 0.000 & 0.075 & 0.030 & 4.58 & $<0.0001$ \\
Geometric mean & 0.943 & 0.915 & 0.944 & 0.883 & 0.860 & 0.931 & 0.921 & 20.65 & $<0.0001$ \\
$\quad$ diameter & & & & & & & & & \\
Surface area & 0.944 & 0.918 & 0.944 & 0.884 & 0.863 & 0.932 & 0.924 & 9.66 & $<0.0001$ \\
Volume & 0.914 & 0.864 & 0.916 & 0.824 & 0.832 & 0.907 & 0.902 & 5.39 & $<0.0001$ \\
\hline
\end{tabular}

$\mathrm{L}: \mathrm{W}=$ length:width; $\mathrm{L}: \mathrm{T}=$ length:thickness; $\mathrm{W}: \mathrm{T}=$ width:thickness.

Table 4. Comparison of coefficients of determination for kernel surface area or geometric mean diameter as functions of kernel weight, with and without effect of harvest year, for 'Nonpareil' almond kernels $(\mathrm{n}=2347)$ harvested during seven consecutive seasons in Parlier, CA, 2003-2009.

\begin{tabular}{lccc}
\hline & \multicolumn{2}{c}{ Harvest yr effects in model } & Difference between \\
Response variable & Present & Absent & \\
\hline Surface area & 0.955 & 0.943 & 1.26 \\
Geometric mean diameter & 0.953 & 0.939 & 1.47 \\
\hline
\end{tabular}

relationship that best described the observed variability across multiple harvests. Pooled data across harvest years denoted $\left(R^{2}=\right.$ $0.943, F$ ratio $=39,116.49, P<0.001)$ the following linear relationship between kernel surface area $\left(\mathrm{mm}^{2}\right)$ and kernel weight $(\mathrm{g})$ :

Kernel surface area

$$
=305.91 * \text { kernel weight }+215.18
$$

Confidence intervals were calculated to establish the range in which any 'Nonpareil' kernel's surface area could be expected based on its weight:

Upper 95\% CI: Kernel surface area

$$
=321.21 * \text { kernel weight }+225.93
$$

$$
\begin{aligned}
& \text { Lower 95\% CI: Kernel surface area } \\
& =290.62 * \text { kernel weight }+204.42
\end{aligned}
$$

in which CI indicates confidence interval.

\section{Discussion}

Almond kernels resemble an irregular prolate spheroid with a specific degree of flattening parallel to the longitudinal cotyledonary axis as determined by varietal character. Interannual variability in kernel dimensions leads to differences in dimensional ratios and other descriptive variables based on kernel dimensions. Our study revealed significant interannual variation in all measured characteristics (Table 1). Significant year-to-year variability in kernel flavonoid and phenolic acid content has also been reported recently in the Nonpareil cultivar (Bolling et al., 2010). These observations are not surprising given that Valverde et al. (2006) reported variation in kernel characteristics of 'Guara' almonds that depended on harvest year and irrigation regime. Ultimately, this suggests that the size and shape of almond kernels as well as specific nutritional qualities are a function of the specific cultivar and the environment in which it is grown. As a result, simple dimensional measures are insufficient for kernel charac- terization and a thorough analysis of the relationships between kernel dimensions will be required to describe adequately the kernels of a single variety.

For 'Nonpareil', which commands a premium price, variation in kernel characteristics resulting from environmental factors could be problematic because consumers expect a consistent product under the Nonpareil marketing category. This is similar to the problem faced by the wine grape industry. Specifically, wine consumers expect wines to possess characteristics specific to the variety of grape used to produce the wine. However, variation in climatic conditions between years can significantly affect secondary metabolite production, sugar content, and vegetative vigor of some grapes (Guidoni et al., 2008). As a result of this year-to-year variability, the varietal character of wines produced from successive harvests is often masked (Arozarena et al., 2000). The development of a varietal standard for 'Nonpareil' that accounts for annual environmental variability and distinguishes it from other California almond cultivars would benefit both consumers and almond handlers by ensuring consistent raw product quality.

Kester and Asay (1975) proposed using kernel weight, kernel length, kernel length: width ratio, and kernel thickness to describe and distinguish almond kernel samples. However, comparison of mean values for each of these measures among years indicated that they varied by as little as 1.1-fold (kernel width) to as much as 1.38 -fold (kernel weight) between years (Table 1). As a result of this interannual variation, additional analyses will be required to distinguish almond kernel samples. Regression analyses are one possible solution to this problem because they take into account changes in kernel size. Regressions of kernel weight on multidimensional measures (kernel geometric mean, kernel surface area, kernel volume) provided a better fit to the data than regression of kernel weight on linear kernel dimensions (kernel length, width, thickness). These relationships were affected by harvest year (Table 3), but the effect was small
(Table 4). Thus, we found that 'Nonpareil' samples could be best described using the linear equation describing the relationship between kernel weight and kernel surface area.

Our results describe variability in characteristics of 'Nonpareil' almonds, but no varietal standard for 'Nonpareil' is claimed based on the results described here. Although we might expect similar variability in 'Nonpareil' kernel samples obtained from different growing regions, the overall phenotypic stability of this old cultivar is apparent given its consistent market share as reported by handlers and acreage predominance throughout the growing regions in California. Clearly, when kernels of other almond cultivars are evaluated in a similar fashion, significant linear equations can be constructed to describe the relationship between kernel size and kernel surface area. With adequate kernel sampling, comparisons can be made between regression slopes of 'Nonpareil' and other cultivars to determine similarities or differences in rates of change. Regression slope might be one of many single variables used in a multivariate analysis that could objectively distinguish a 'Nonpareil' kernel sample from kernel samples of other cultivars.

Sample sizes in our study ranged from small (30 in 2003) to large (862 in 2008). The International Union for the Protection of New Varieties of Plants (UPOV) provides recommendations in the administration of tests to determine varietal distinctness, homogeneity, and stability. For almond, UPOV recommends a sample size of at least 30 when evaluating traits pertinent to fruits and/or kernels (UPOV, 1978). Our results agree that a sample size of 30 provides a reasonably accurate estimate of the mean and variance for a variety of kernel measurements (Fig. 1). In fact, our resampling procedure suggests that there is diminishing return for having sample sizes larger than 30 and that a sample size of 20 may even be acceptable. These results are further corroborated by the fact that values obtained in 2003, the year with the smallest sample size $(n=30)$, yielded averages that were similar with averages from other years when sample sizes were larger.

\section{Conclusions}

Interannual variability of 19 carpological characters of 'Nonpareil' almond was examined in a California orchard from 2003 through 2009. There was significant interannual variation in all examined characters. Of the gravimetric and dimensional variables evaluated, kernel weight correlated best with the multidimensional variables geometric mean diameter, surface area, and volume. To further describe 'Nonpareil' kernels, regression analyses were completed that examined the relationships of kernel dimensions, kernel dimensional ratios, and multidimensional characteristics with kernel weight. Of the examined regressions, kernel surface area regressed on kernel weight accounted for the most variability in sampled 'Nonpareil' kernels. Pooled data from the 7 consecutive harvest years were used to develop the linear equation that best 
explained the observed variability between these characters across multiple harvests.

\section{Literature Cited}

Aktas, T., R. Polat, and U. Atay. 2007. Comparison of mechanical properties of some selected almond cultivars with hard and soft shell under compression loading. J. Food Process Eng. 30:773-789.

Anon. 1997. United States standards for grades of shelled almonds. US Department of Agriculture, Agricultural Marketing Service, Fruit and Vegetable Division, Fresh Products Branch § 51.2116.

Anon. 2009. Almond Board of California 2009 Almond Almanac. Mar. 2010. <http://www.almondboard. com/AboutTheAlmondBoard/Documents/2009Almond-Board-Almanac.pdf $>$.

Arozarena, I., A. Casp, R. Marín, and M. Navarro. 2000. Multivariate differentiation of Spanish red wines according to region and variety. J. Sci. Food Agr. 80:1909-1917.

Asaii, W.K., W.C. Micke, D.E. Kester, and D. Rough. 1996. The evaluation and selection of current varieties, p. 52-60. In: Micke, W.C. (ed.). Almond production manual. University of California, Davis, CA.

Bolling, B.W., G. Dolnikowski, J.B. Blumberg, and C.-Y. Oliver Chen. 2010. Polyphenol content and antioxidant activity of California almonds depend on cultivar and harvest year. Food Chem. 122:819-825.
Clydesdale, F.M. 1991. Color perception and food quality. J. Food Qual. 14:61-74.

Dangl, G.S., J. Yang, D.A. Golino, and T. Gradziel. 2009. A practical method for almond cultivar identification and parental analysis using simple sequence repeat markers. Euphytica 168 : $41-48$.

García-López, C., N. Grandé-Teruel, V. Berenguer-Navarro, J.E. García-García, and M.L. Martín-Carratalá. 1996. Major fatty acid composition of 19 almond cultivars of different origins. A chemometric approach. J. Agr. Food Chem. 44:1751-1755.

Guidoni, S., A. Ferrandino, and V. Novello. 2008 Effects of seasonal and agronomical practices on skin anthocyanin profile of Nebbiolo grapes. Amer. J. Enol. Viticult. 59:22-29.

Kester, D.E. and R. Asay. 1975. Almonds, p. $387-$ 419. In: Janick, J. and J.N. Moore (eds.). Advances in fruit breeding. Purdue University Press, West Lafayette, IN.

Ledbetter, C.A. and D.E. Palmquist. 2006a. Comparing physical measures and mechanical cracking products of 'Nonpareil' almond [Prunus dulcis (Mill.) D.A. Webb.] with two advanced breeding selections. J. Food Eng. 76 : 232-237.

Ledbetter, C.A. and D.E. Palmquist. 2006b. Degradation of almond pellicle color coordinates at different storage temperatures. Postharvest Biol. Technol. 40:295-300.
Manly, B.F.J. 1991. Randomization, bootstrap and Monte Carlo methods in biology. Chapman and Hall, New York, NY

Martín-Carratalá, M.L., M.S. Pratts Moya, N. Grané Teruel, and V. Berenguer Navarro. 2002. Discriminating significance of the free amino acid profile in almond seeds. J. Agr. Food Chem. 50:6841-6846.

Özgüven, F. and K. Vursavuş. 2005. Some physical, mechanical and aerodynamic properties of pine (Pinus pinea) nuts. J. Food Eng. 68:191196.

Sánchez-Pérez, R., E. Ortega, H. Duval, P. MartínezGómez, and F. Dicenta. 2007. Inheritance and relationships of important agronomic traits in almond. Euphytica 155:381-391.

Spiegel-Roy, P. and J. Kochba. 1981. Inheritance of nut and kernel traits in almond (Prunus amygdalus Batsch). Euphytica 30:167-174.

UPOV. 1978. Almond. Guidelines for the conduct of tests for distinctness, homogeneity and stability. TG/56/3. Apr. 2010. <http://www.upov.int/en/ publications/tg-rom/tg056/tg_56_3.pdf>.

Valverde, M., R. Madrid, and A. García. 2006. Effect of the irrigation regime, type of fertilization, and culture year on the physical properties of almond (cv. Guara). J. Food Eng. 76:584 593.

Wood, M.N. 1937. Almond culture in California. California Agricultural Extension Service. Circular 103. 